\title{
Discussions on Proposed Boost Inverters in Integrated Construction
}

\author{
- Pursuit for simple power conditioner -
}

\author{
Keiju Matsui ${ }^{a^{*}}$, Eiji Oishi ${ }^{a}$, Yasutaka Kawata ${ }^{\text {a }}$, \\ Mikio Yasubayashi ${ }^{\mathrm{b}}$, Masayoshi Umeno ${ }^{\mathrm{b}}$, Hideo Uchida $^{\mathrm{b}}$, Masaru Hasegawa ${ }^{\mathrm{b}}$ \\ ${ }^{a}$ Minna-denryoku,Inc. Setagaya Monozukuri Gakko, 210 Setagaya154-0001, Japan \\ ${ }^{\mathrm{b}}$ Chubu University Kasugai 487-8501, Japan
}

\begin{abstract}
Photovoltaic power generations (PVG) have been widely accepted and applied. Various power conditioning systems including boost-inverters have been also studied by many researchers. In general utilization, the actual application of such solar panels are almost installed on top of the roof of isolated house. Some residents living in the apartment house, however, have fair desires to contribute for energy saving due to natural energy generation. The generating power in such case is fairly reduced, so the system construction should balance to the reduced power. Thus, it is necessary to improve the construction toward simple one. In this paper, in order to give a reply, simple and concise power conditioner is proposed, especially integrated inverter. Considering fairly reduced generating power and narrow space of installation, the system constructions should be compact. The circuits which gratifies their characteristics are presented and examined.
\end{abstract}

Keywords: PVG, boost inverter, boost chopper, full-bridge, half-bridge, inverter.

\section{Introduction}

Preserving the developments of economy, science and technology with conquering the problems against environmental conservation is an important assignment for humanity of the world. The usage of the renewable energies is expected to be able to mitigate such problem, which is an important means [1,2]. Among them, photovoltaic power generation have various advantages such as usage of inexhaustible and unpolluted sunlight, which is advantageous for maintenances, easily construction from small power to large one, and can be installed in various location including city center. In such a way, this power generation system is a promising one with bright future.

As a utilization form, utility interactive power generation system has been accepted and spread widely. In such system, it is necessary to install the chopper, where the lower voltage of solar cell should be boosted to suitable voltage, and inverter where the $\mathrm{dc}$ power of boosted voltage should be converted to ac power in the power system $[3,4]$.

The power conditioners-PCS including inverter have been presented in various systems so far $[5,6]$. However, it is necessary to reduce the cost even more. It is said that the system is approaching to an ideal ones with respect to efficiency and construction strategy, but that cost would prevent wide spread if there is no public financial support. In such discussions, there are many subjects to be solved to utilize the PV power in utility interactive power generation. Even more, various safeguard equipment required according to regulations make the cost increase Thus, it is required to obtain even lower cost PCS. [7-9] In an extremely lower capacity PCS as discussed in this paper, a way of handling would be different compared to typical power range. In such case of reduced generating power, quantities of reversed power to the power system would be small, where another mitigated regulation or deregulation would be approved. Thus, in such PV power generation systems, there are so many subjects to be resolved[10].

Under such circumstances accepted by electrical utility industry as recognitions and assessments for renewable energy, spread of application is strongly sustained by financial supports of public organizations, and a lot of consumers are hoping to install such PV power generation system. For ordinary homes, installation of solar panel is restricted to house having roofs. However, a lot of residents living in the apartment house also wish to install the PV panel under considering resource conservation and such public financial support. In the present situation, however, there is no scheme to perform these requirements. The authors have been studied in a series of the small power PV system [11,12]. In this paper, some simple PCS systems, especially the components like inverters and chopper are integrated toward simple construction, which are presented and discussed.

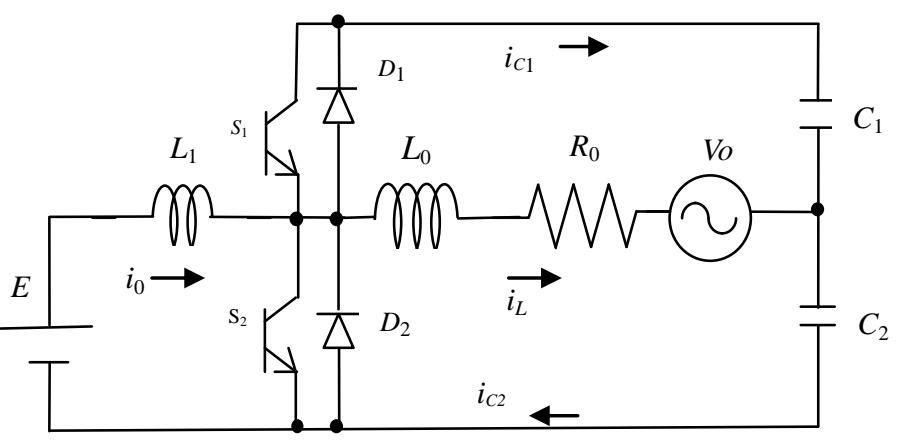

Fig.1. Initial proposed boost inverter. 


\section{Initial Proposed Boost Inverter Using Half-bridge Circuit}

\subsection{Summary of operation}

Fig.1 shows an example of initial PCS (Power Conditioners) which is intended for compact construction by the half bridge circuit. The single phase or three phase constructions using full-bridge circuit would be possible for various applications, but the explanation is performed for fundamental half-bridge circuit. In the conventional inverter, the boosted output voltage from boost converter is used. In this PCS, however, the supply voltage is given to the midpoint of inverter leg through current limiting inductor as shown.

The operation is performed as follows; for the switches $S_{1}$ and $S_{2}$ which are operating for normal load of $L_{o}$ and $R_{0}$ or ac power source $\mathrm{V}_{\mathrm{o}}$, the sinusoidal signals are given as usual. In this control strategy, for example, of natural sampling method (triangular-sinusoidal wave comparison method), as the average turn-on ratio is given by $\alpha=0.5$, the dc link voltage of inverter is boosted by two times which can be calculated from the boosting theory in boost converter. As a result, the input dc power supply voltage can be boosted by two times. In such a way, $2 E$ voltage can be obtained across the inverter capacitors, where $E$ is input dc supply voltage. $S_{2}$ plays also a role of the function of boost converter switches. By means of using merely single inverter construction, a novel inverter construction having boosted voltage capability can be obtained. The dc link voltage of inverter is given by

$$
\begin{aligned}
& V_{I}=E /(1-d) \\
& \text { where } \quad d: \text { the average value of duty ratio } \\
& E: \text { Input power supply voltage }
\end{aligned}
$$

Assuming the sinusoidal and triangular wave comparison method, regardless of the modulation factor (ratio of sinusoidal amplitude / triangular amplitude), average value of conduction factor becomes $d=0.5$. Consequently, inverter dc link voltage becomes $V_{I}=2 E$.

For the input dc supply voltage of $E$, by means of principle of the proposed circuit, the dc link voltage is given by $V_{\boldsymbol{C} \boldsymbol{I}}+V_{\boldsymbol{C} 2}=2 E$. The peak voltage $V_{o}$ of load becomes $V_{o}=2 E / 2$.

\subsection{Characteristics of operation}

The input current is varying with output load frequency. The reason can be explained by theoretical equation where the commercial frequency is applied across the current limiting inductor. The average value of this input current is injected as average power. This value of input current has nothing to do with the load current amplitude. That is to say, the ac component with output frequency is superimposed to the constant dc current which is average value of instantaneous input current. From such reasons, input ac current component is limited by small limiting inductor.
On the next stage of comparison with $i_{c 1}$ and $i_{c 2}$, the operation mechanism of presented PCS can be confirmed and perceived. The upper side $i_{c l}$ represents the charging current from input current of dc source and the load current from inductive load at $S_{2}$ turned off and $S_{1}$ turned on, which is shown in the positive side. That is,

$$
\begin{aligned}
& \text { When } S_{1} \text { turned-on, }\left(S_{2} \text { turned-off }\right), \\
& i_{c 1}=i_{o}(\text { charge current })+i_{L}(\text { load current }) \\
& i_{c 2}=i_{o}(\text { charge current })
\end{aligned}
$$

When $S_{2}$ turned-on, ( $S_{1}$ turned-off),

$$
\begin{aligned}
i_{c 1} & =0 \\
i_{c 2} & =i_{L}(\text { load current })
\end{aligned}
$$

In upper side loop of current $i_{c l}, i_{o}$ (charge current) $+i_{L}$ (load current) and $i_{c l}=0$ are repeated periodically. Consequently, the current flows through the envelope of $i_{o}+i_{L}$. On the other hand, in lower side loop of $i_{c 2}, i_{o}$ (charge current) and $i_{L}$ (load current) are repeated periodically, where current flows with envelope of $i_{o}$ and $i_{L}$. Such operation is confirmed and perceived.[10]

In this circuit, regenerating operation from ac load power source to input dc source, can be realized in which bilateral control is performed successfully without additional considerations in the circuit construction and the control circuit.

\section{Proposed Boost Inverter Using Integrated Full-bridge Construction}

\subsection{Summary of operation}

In order to realize another boost inverter using single stage, the front end of switching devices $S_{1}$ and $S_{2}$ of inverter are turned on simultaneously to make function as the boost chopper. At this period of simultaneous operation of $S_{1}$ and $S_{2}$, the power due to the short circuit is injected into the input inductor.

By means of this single stage, both functions of boosting and dc to ac conversion are intended to realize. The method to realize single stage circuit configuration will be described as follows;

In Fig.4, The terminals A, B, C and D are connected the designated same terminals of Fig.4(b). By using full bridge circuit, where the whole portion constitutes inverter circuit. Partially, the boost chopper is constituted by $S_{1}$ and $\mathrm{S}_{2}$. By means of single stage, inverter function having boosted voltage can be performed. When the chopper is operating, the electric charge of $\mathrm{C}_{1}$ or $\mathrm{C}_{2}$ is provided into the load through switching device $\mathrm{S}_{3}$ or $\mathrm{S}_{4}$. In order to prevent discharge of $\mathrm{C}_{1}$ or $\mathrm{C}_{2}$ through short circuit of $\mathrm{S}_{1}$ and $S_{2}$, the diodes $D_{5}$ and $D_{6}$ are equipped as preventing the short circuit. There are two operation modes, where one is simultaneous conduction mode of $S_{1}$ and $S_{2}$ and other is load powering mode due to inverter operation. By means of the smoothing capacitors $\mathrm{C}_{1}$ and $\mathrm{C}_{2}$, the power is injected into the load as inverter operation. When $S_{1}$ or $S_{2}$ is turned off, the dc power supply and the charged inductor provide the power into the smoothing capacitors $\mathrm{C}_{1}$ and $\mathrm{C}_{2}$ 
through the reverse current preventing diodes $\mathrm{D}_{5}$ and $\mathrm{D}_{6}$.

As just mentioned, the chopper operation and inverter operation due to $S_{1}$ and $S_{4}$, or $S_{2}$ and $S_{3}$ are performed simultaneously by means of single stage. The diodes $D_{5}$ and $D_{6}$ are preventing the short circuit in charging mode due to the boost chopper. By means of single stage, inverter function having boosted voltage can be performed successfully.

\subsection{Operational waveforms}

Fig.3 shows operational waveforms for the proposed boost inverter. In order to make clear the operation, the carrier triangular frequency is given by lower one and unity power factor of zero load inductance that is $L_{o}=0$. When one switch is turned on for half period, the other signal is periodically turned on and off in PWM patter.

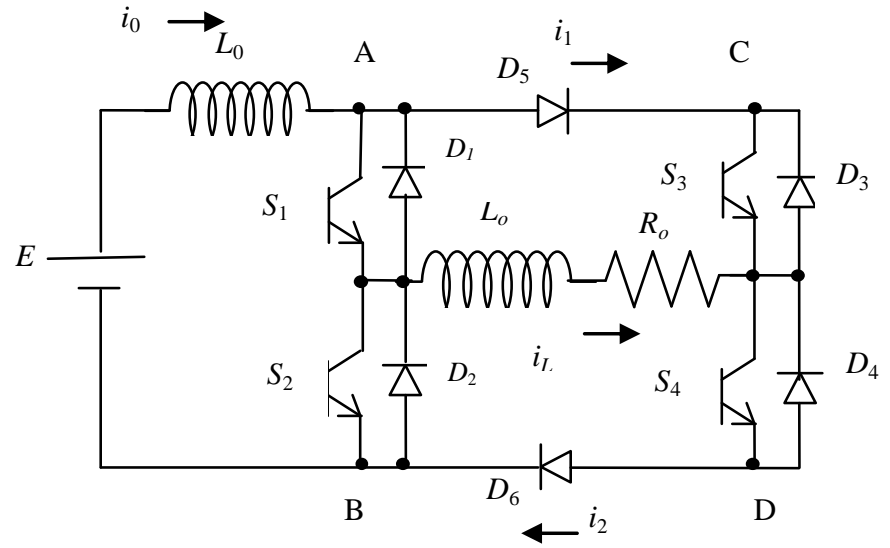

(a)

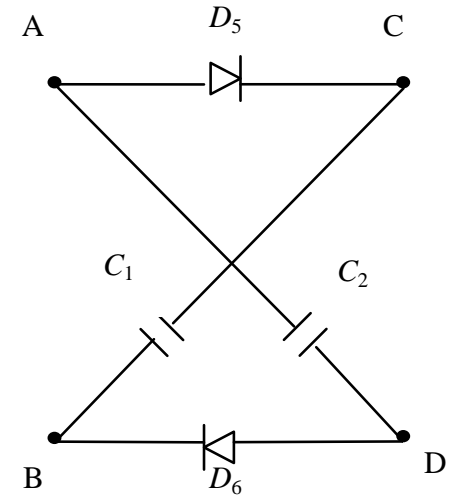

(b)

Fig.2. Circuit configuration of another boost inverter.

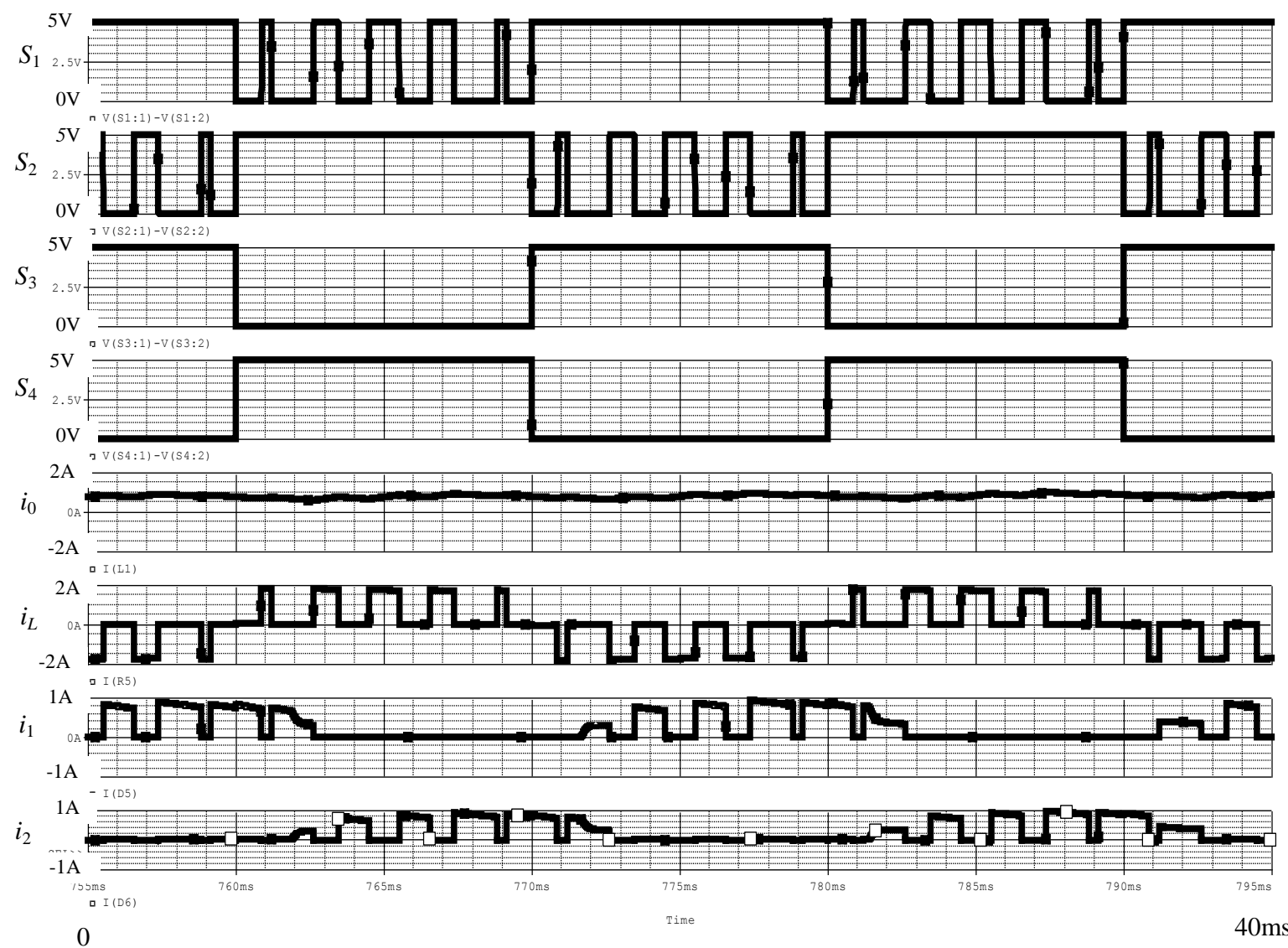

Fig.3. Waveforms.(at $L_{o}=0$ ) 
The diode currents are flowing through $D_{5}$ or $D_{6}$ alternately in PWM pattern. When the polarity of load current changes, both diode currents charge both capacitor. As a result, currents become in half as presented. In practical operation, the PWM switching frequency is given by higher one, and additional small inductance is equipped, so load current becomes almost sinusoidal waveform.

\subsection{Control of PWM waveforms}

\section{(a) single PWM control method}

Fig.3 shows simulated results, where the load does not include inductive component $\left(\mathrm{L}_{\mathrm{o}}=0\right)$ in order to make the circuit mechanism clear. That is, if this configuration is operated satisfactorily according to fairly high power factor load.

In this first single signal method, the PWM signal for $S_{1}$ and $S_{2}$ is given by simple uni-polar triangular carrier signal, which is compared to sinusoidal modulation wave to obtain the practical signal.

As the period when both switches $S_{1}$ and $S_{2}$ are turned on simultaneously, is given as the duty cycle $=\mathrm{d}$ of boost chopper, it is necessary to obtain the conduction period that is given by the average value. That is,

$$
\begin{aligned}
d & =(1 / \pi) \int \alpha \sin \theta \mathrm{d} \theta \\
& =2 \alpha / \pi \quad \ldots \ldots \ldots \ldots \ldots \ldots \ldots(2)
\end{aligned}
$$

The equation (1) is substituted into the boost ratio that is ratio of input and output voltage.

$$
E_{2} / E_{1}=1 /(1-d)=\pi /(\pi-2 \alpha) \cdots \cdots \cdots \cdots(3)
$$

The maximum boost ratio is given by at $\alpha=1$. That is,

$$
\left(\mathrm{E}_{2} / \mathrm{E}_{1}\right)_{\max .}=1.47 \cdots \cdots \cdots \cdots \cdots \cdots(4)
$$

(b) double PWM control method

In this section, another PWM control method is to be described where both S1 and S4 are controlled over this period for obtaining the simple PWM signal. Another signal $S_{2}$ corresponds to signal $S_{3}$. The control of $S_{1}$ is performed by bipolar carrier triangular wave. The output pulse over half period can be represented by switching function $S_{\mathrm{W} 1}(\theta)$.

$$
\mathrm{S}_{\mathrm{W} 1}(\theta)=1 / 2+(1 / 2) \cdot \alpha \sin \theta \cdots \cdots \cdot(5)
$$

On the other hand, the corresponding $\mathrm{S}_{4}$ signal is also represented by switching function, that is,

$$
\mathrm{S}_{\mathrm{W} 4}(\theta)=1 / 2-(1 / 2) \cdot \alpha \sin \theta \cdots \cdots \cdot(6)
$$

The applied voltage across the load becomes,

$$
V_{\mathrm{o}}=E_{2} \cdot\left(\mathrm{S}_{\mathrm{W} 1}(\theta)-\mathrm{S}_{\mathrm{W} 4}(\theta)\right) \cdot \alpha \sin \theta \cdots(7)
$$

On the other hand, S2 has the same period when the signal for $S_{1}$ is given as the boosted operation at the same time. That is represented by switching function as follows;

$$
\mathrm{S}_{\mathrm{W} 2}(\theta)=1 / 2 \ldots \ldots \ldots \ldots \ldots \ldots \ldots \ldots(8)
$$

$E_{2} / E_{1}=1 /(1-\mathrm{d})=2.0 \cdots \cdots \cdots \cdots \cdots \cdots(9)$

The boosting ratio is given by this equation. As comparison with single PWM control, boosting ratio becomes fairly larger.

\subsection{Operation of load capacitor}

By means of current limiting inductor, the input current is kept almost constant value. That current is divided into th input front inverter leg and load capacitor. The operation can be executed as follows. When the switch $\mathrm{S} 1$ is turned on, where $\mathrm{S} 2$ is turned on simultaneously, the input inductor is energy-stored by input supply for boost operation. That is,

$$
\begin{aligned}
& \text { When } S_{1} \text { turned-on, }\left(S_{2} \text { also turned-on }\right) \\
& i_{o}=i_{S}(\text { short circuit current }) \\
& i_{L}(\text { load current })=i_{C 2}(\text { capacitor current })
\end{aligned}
$$

$$
\begin{aligned}
& \text { When } S_{1} \text { turned-off, } \\
& \qquad \begin{array}{l}
i_{o}=i_{C 2}(\text { capacitor current }) \\
i_{L}=0
\end{array}
\end{aligned}
$$

In the figure, according to the above description, the input supply current is delivered into short circuit and load capacitors alternately. In the transition period, however, as the input current flows through both load capacitors, the overlap period appears as shown.

\section{Conclusions}

A novel inverter construction termed "Boost inverter" is proposed, whose dc voltage can be boosted by itself without much modification of the circuit configuration. However, the conventional boost inverters are well known as various articles [13-16]. Our proposed method is different from such one. From such reasons, the mentioned configuration is sometimes called "L source inverter", because having input inductor.

In the proposed two types construction, each characteristic can be described with different one.

For initial proposed construction, in satisfactory characteristic is that there is no consideration for signals for boost chopper. In addition, there is no special consideration for changing from powering to regenerating. The conversion operation from powering to regenerating can be altered naturally. In such natural bilateral operation, it can be performed as it is without special configuration and accompanied control circuitry.

In single phase or three phase full-bridge construction, the input power supply could be also connected to each center terminal of inverter legs through inductor of itself, that is double inductors for single phase or three inductors for three phase. In this initial method, however, input inductor size becomes larger one. So, this type is suitable for the constant current source supply.

For secondly proposed construction, The construction becomes a little complicated as compared. However, input inductance becomes much smaller for last PWM strategy. It would be applied to reduced power application. Without changing the configuration, regenerative operation to input side cannot be realized. When it is necessary to realize such capability of power regeneration, some devises 
should be installed such as independent boost chopper.

\section{Acknowledgements}

This research is mostly supported by a grant of the NEDO (New Energy and Industry Technology Development Organization). We would like to express our appreciation to who it may concern about this project.

\section{References}

(1) Yan Hu, Keiju Matsui, Takashi Sugiyama, Kenji Ando and Isamu Yamamoto," $3 \mathrm{~kW}$ Utility-interactive Power Conditioning System using Forward Converter", Proceedings of Static Power Converter Meeting in IEEJ, SPC-94-89,pp.49-56,(1994-10)

(2) Chihiro Okado. : "Development of inverter for photovoltaic generation". Proceedings of 10th Photovoltaic Generation System symposium, Tokyo, Japan, 1993, pp.411 (in Japanese)

(3) Sakae Shibasaki, Isao Takahashi, Shinzo Sakuma, Noriyasu Mimura, Yoshihiko Asano, "Small Quantity and Thin Type 200W Inverter for Photovoltaic Systems", Proceedings of National Convention in IEEJ,739,pp.4-50-51

(4) Khoder, K.Al-Haddad and V.Rajagopalan: "Innovative utility-interactive de to ac power conditioning system'. Conference Record of 1985 IEEE IAS Annual Meeting, Toronto, Canada, 1985, pp.1151-1155

(5) Keiju Matsui, Eiji Oishi, Y.Kawata, M.Yasubayashi, M.Umeno, H.Uchida, M.Hasegawa: "Simple and Concise Photovoltaic Power Generation Systems installed in Verandas of Apartment House",Journal of Japan IE, Vol.94, No.6, pp.456-460, 2015-6

(6) Eiji Oishi, Keiju Matsui,Masayoshi Umeno, Mikio Yasubayashi, Hideo Uchida, Yasutaka Kawata,'Simple and Sophisticated Maximum Power Tracker for PV Power Generator", 2014 International Conference on Information Science, Electronics and Electrical Engineering, IEEE-ISEEE,Sapporo, pp.1815-1820 (2014-4)

(7) Keiju Matsui, Eiji Oishi, Yasutaka Kawata, Mikio Yasubayashi, Masayoshi Umeno, Hideo Uchida, Masaru Hasegawa," Proposal of Boost - Inverter Having Voltage Doubler Capability", The $19^{\text {th }}$ ICEMS (2016-11)

(8) Edit: Ohmsha,"Guide Book of Technical Standard Concerning Electrical Equipment", 1st edit., pp.52-63, pp.376-382, 2013

(9) Technical Committee for Customer Equipment, edit., "Indoor Wiring Regulation - electrical code for customer equipment", Electric Association of Japan, pp.858-860, 2012-3

(10) Keiju Matsui, Eiji Oishi, Yasutaka Kawata, Mikio Yasubayashi, Masayoshi Umeno, Hideo Uchida,Masaru Hasegawa: "Pursuit of Simple PCS for Photovoltaic Power Generation - Optimum Waveforms", IEEE-Intelec2015, pp.915-920, 2015.10
(11) Keiju Matsui, Eiji Oishi, Yasutaka Kawata, Mikio Yasubayashi, Masayoshi Umeno, Hideo Uchida, Masaru Hasegawa, "Discussions on Photovoltaic Power Conditioners Installed in Veranda of Apartment Houses, ACSEE 2014, The Asian Conference on Sustainability, Energy and the Environment, Osaka, pp.455-466 (2014-6)

(12) Keiju Matsui, Eiji Oishi, Yasutaka Kawata, Mikio Yasubayashi, Masayoshi Umeno, Hideo Uchida, Masaru Hasegawa,"Simple and Concise Photovoltaic Power Generation System Installed in Veranda", The IEEE- APCCAS 2014, the 12th of the biennial Asia Pacific Conference on Circuits and Systems, pp.567-570 (2014-11)

(13) Keiju Matsui, Eiji Oishi, Yasutaka Kawata, Mikio Yubayashi, Masayoshi Umeno, Hideo Uchida, Masaru Hasegawa: 'The Pursuit for Simple Power Conditioner of Photovoltaic Small Power Generation“, IEEE Industrial Electronics Conference, Yokohama, Proceedings of IEEE-IECON 2015, pp.556-561, 2015-11

(14) R.O.Caceres and Barbi, "A boost DC-AC converter; analysis, design and experimentation," Trans. On Power Electronics,vol.14, pp.134-141,1999

(15) Minsoo Jang and Vassilios G. Agelidis; "A minimum power processing stage fuel-cell energy system based on a boost-inverter with a bidirectional backup battery storage", Trans. On Power Electronics, vol.26, pp.1568-1577,2011

(16) Minsoo Jang,Takyun Kim,Vassilios G. Agelidis; "Design and Implementation of a $200 \mathrm{kHz}$ Single-Phase Boost-Inverter Using Silicon Carbide Semiconductors",Proceedings of IEEE IECON 2015 Yokohama, pp.2241-2246, 2015-11 